\title{
Rapid Diagnosis of Chlamydial Infection in Young Women at Reproductive Age
}

\author{
Ismaeil M. Abdulkahar Mammani ${ }^{1}$, Amer A. Goreal ${ }^{1}$, Waleed J. Omer ${ }^{1}$ and Sabeeha A. Mansor ${ }^{2}$ \\ 1. Department of Microbiology, College of Medicine, University of Duhok, Kurdistan Region, Iraq \\ 2. Microbiology Laboratory, Azadi Teaching Hospital, Duhok, Kurdistan Region, Iraq
}

\begin{abstract}
Aim: To determine the seroprevalence of Chlamydia trachomatis in a group of randomly selected pregnant women, using the ELISA IgG and IgM assays. The study included 88 married women whose age range was 19-38 years. This study was conducted in the Microbiology lab at Azadi Teaching Hospital from February 2009 to Dec 2009 in Duhok city. Two endocervical swabs were taken from each woman, the first to be examined as wet preparation stain to find bacteria, RBCs, epithelial cells, monilia, Trichomonas vaginalis, the second swab was used to examine for the presence of Chlamydia trachomatis antibodies by ELISA anti Chlamydia specific IgM, and anti Chlamydia specific IgG. The wet preparation examination of the swabs revealed Trichomonas vaginalis in 4 patients. Gram's stain revealed the presence of Gram positive Streptococci in 10 patients, Gram negative bacilli in 6 patients, and no bacteria was found in 45 patients. ELISA test to detect anti Chlamydia Antibodies (IgM), all the 88 examined women were negative. ELISA test to measure the anti Chlamydial antibodies (IgG) detect chronic infections and the results showed ten positive cases, 10/88 (11.3\%). The age group of eight out of ten IgG positive cases is between 21-30 years old, for the remaining two cases is between 31-40 years old. Five of the IgG positive cases had history of ectopic pregnancies; another two had history of acute conjunctivitis in their newborn babies, while the remaining three had chronic infection and discharge. The results of this study revealed that the commercially available rapid test is of no value in detection of Chlamydia in endocervical swabs. The trend should be toward the substitution of this test by the direct immunoflourescence test. The nucleic acid amplification test which is highly sensitive should be used in future studies to detect the prevalence of Chlamydia infection.
\end{abstract}

Key words: Chlamydia trachomatis, rapid test, ELISA test, cervical swab.

\section{Introduction}

Chlamydia is small gram-negative eubacteria that grow intracellular. There are four species (Chlamydia trachomatis, C. psittaci, C. pneumonia and C. pecorum), the first three of which have been associated with various human diseases involving particular populations. Chlamydia trachomatis is a common bacterial cause of sexually transmitted disease worldwide and is responsible for high levels of morbidity [1, 2].

C trachomatis serovars $\mathrm{A}, \mathrm{B}, \mathrm{Ba}$ and $\mathrm{C}$ are associated with endemic trachoma, which is the most common preventable form of blindness in certain parts of the Mediterranean and Middle East. Serovars L1, L2

Corresponding author: Waleed J. Omer, $\mathrm{PhD}$, research field: parasitology.E-mail: waleed_68@yahoo.co.uk. and L3 are associated with lymphogranuloma venereum (LGV) in tropical settings. Serovars D through $\mathrm{K}$ cause nongonococcal urethritis and epididymitis in men, Reiter's syndrome or proctitis, conjunctivitis in both men and women, and cervicitis, urethritis, endometritis, salpingitis and perihepatitis in women [3]. Between one-half and two-thirds of chlamydial infections in men and women may be asymptomatic and remain undiagnosed and untreated. In women, this may lead to late sequelae such as endometritis, salpingitis, pelvic inflammatory disease, ectopic pregnancy or tubal factor infertility. $C$ trachomatis in the cervix may be transmitted to a neonate during vaginal delivery, resulting in conjunctivitis and neonatal pneumonia [4].

It is also the major cause of genitourinary infection in developed countries [5]. Chlamydia trachomatis can 
cause infections of the cervix, urethra and upper genital tract in women, infections of the urethra and epididymis in men, and the cause of conjunctivitis and pneumonia in newborns [6]. It has been estimated that some $10-40 \%$ of inadequately treated women with chlamydial cervicitis develop pelvic inflammatory disease (PID) which is an important cause of infertility, chronic pelvic pain, ectopic pregnancy, and adverse outcome of pregnancy $[7,8]$. In a majority of women, infections are asymptomatic and thus require laboratory testing for diagnosis. For this reason, laboratory screening of young women has been considered the cornerstone of Chlamydia screening programmes. These programmes have been associated with subsequent reductions in the prevalence of Chlamydia in a given population, as well as reduced the incidence of PID among the women screened [9].

The most sensitive method for the diagnosis of a genital C. trachomatis infection was, until recently, based on tissue culture of the microorganism on McCoy cells, and was regarded as the "gold standard". However, there are several disadvantages of cell culture [10, 11]. These have led to the search for alternative techniques for the detection of $C$. trachomatis. Besides the development of antigen detection techniques, such as direct fluorescent-antibody tests and enzyme immunoassays $[12,13]$, nucleic acid amplification techniques have been developed [14, 15].

Nucleic acid amplification tests (NAAT's) have generally been more sensitive than traditional tests. The prevalence of chlamydia genital infection in women varies in different groups and communities. The incidence of $C$. trachomatis infection in asymptomatic unselected pregnant women varies from 4 to $21 \%$ [16].

The aim of the present study was, therefore, to determine the seroprevalence of Chlamydia trachomatis in a group of randomly selected pregnant women, using the ELISA IgG and IgM assays.

\section{Patients, Material \& Methods}

This study was conducted in the Microbiology lab at Azadi Teaching Hospital from February 2009 to Dec 2009. The study was designed to detect the incidence rate of Chlamydia trachomatis infection in the genital tract of married women living in and around Duhok city who were complaining of different clinical symptoms for which they had consulted the gynecologist at Obstetric and Gynecology Department at Azadi teaching hospital in Duhok.

The study included 88 married women their age range was 19-38 years $($ mean $=27.5)$.They were complaining of different symptoms including: dysurea, chronic vaginal discharge, pelvic pain, abortion and ectopic pregnancy. Some of them were visiting the Gynecology department for the first time and for some others was the second or the third visit.

Each woman was asked to answer the questionnaire which included name, age, address, and socioeconomic status, number of live births, abortions, ectopic pregnancies, and history of acute conjunctivitis of their newborns.

(1) Two endocervical swabs were taken from each woman, the first to be examined as wet preparation stain to find bacteria, RBCs, epithelial cells, monilia, Trichomonas vaginalis, then Gram's stain of the swab was done to see the different forms of bacteria inoculating the cervical region.

The second swab was used to examine for the presence of Chlamydia trachomatis antigens by one step Chlamydia test (Precheck, CHLA 341) which is rapid qualitative immunoassay based on immunochromatographic principle. Before specimen collection a swab was used to remove excess mucus from endocervical area and discarded, then another swab with Dacron tip was introduced to the endocervical canal to obtain the epithelial cells which contain Chlamydia, then test was done according to manufacturer instructions.

(2) From each woman participated in this study 5Ml of venous blood was collected by venepuncture and put 
in plain tube for centrifugation at (3000 rpm) and serum was collected and stored at $\left(-20^{\circ} \mathrm{C}\right)$ till used for the following serological tests:

(1) ELISA kit (Novatec immunodignostica GMBH) for qualitative determination of anti Chlamydia specific IgM, which is a marker of acute recent infection.

(2) ELISA kit (Novatec immunodignostica GMBH) for qualitative determination of anti Chlamydia specific IgG, which is marker of chronic infection. Samples are considered positive if the absorbance value is greater than $10 \%$ over cut off value and negative if the absorbance value is lower than $10 \%$ below the cut off value.

\section{Results}

(1) The wet preparation examination of the swabs revealed the following:

- Pus cells from 5-20 cells/ml in 34 patients.

- Trichomonas vaginalis in 4 patients.

- bacteria from 10-30 cells/ $\mathrm{ml}$ in 24 patients.

- Monilia in 5 patients.

- RBCs from 10-30/ml in 15 patients.

- Clear in 30 patients.

Gram's stain revealed the presence of Gram positive Streptococci in 10 patients, Gram negative bacilli in 6 patients, and no bacteria was found in 45 patients.

(2) The rapid test was used to detect Chlamydial antigen from cervical swabs, all the swabs taken from 88 patients were negative:

(3) ELISA tests were used to measure anti Chlamydial antibodies in the serum of these patients and the results were as follows:

- $\quad$ ELISA test to detect anti Chlamydia Antibodies (IgM), all the 88 examined women were negative.

- ELISA test to measure the anti Chlamydial antibodies (IgG) to detect chronic infections and the results showed ten positive cases, 10/88 (11.3\%).

The age group of eight out of ten IgG positive cases is between 21-30 years old, for the remaining two cases is between $31-40$ years old.
Five of the IgG positive cases had history of ectopic pregnancies, another two had history of acute conjunctivitis in their newborn babies, while the remaining three had chronic infection and discharge.

\section{Discussion}

The prevalence of clinical and subclinical infections due to $C$. Trachomatis have been reported as high in both men and women in many countries. The World Health Organization estimated that 89 million cases of C. trachomatis infection occurred worldwide [17]. In the United States, C. trachomatis infections are the most commonly reported bacterial disease, with an estimated 4-5 million cases occurring annually. The sequelae of $C$. trachomatis infections in women, namely pelvic inflammatory disease (PID), infertility and ectopic pregnancy, are the most costly outcome of any sexually transmitted disease.

Neonates usually become infected with $C$. trachomatis during birth. Conjunctivitis, pneumonia, myocarditis, otitis media and other diseases may develop in neonates born to mothers infected with chlamydia $[4,18]$. The results of this study revealed that the prevalence of $C$. trachomatis is $11.3 \%$.

The results of different studies revealed that the prevalence of C. trachomatis infection in pregnant women ranges from 2 to $35 \%$ [19, 20].

A study done by Ghazi [21], revealed that the prevalence of infection in Saudi women was $8.7 \%$, on the other hand Hashemi [22] reported the frequency of CT infection (12\%) among the 20-to-30-year-old women. in a study carried out by Forsey and Darougar [23], $10 \%$ of the females attending gynecology clinics were positive for chlamydia. which is very close to our results.

Rapid test is not sensitive since it couldn't detect any of the 10 positive (IgG) cases. These results should attract the attention of obstetricians not to depend on such a rapid test in the diagnosis of suspected Chlamydial cervicitis. A study done by Sirmatel [24] in southeast Turkey used the direct immunoflourescence 
test as a rapid test to detect Chlamydial antigens in endocervical swabs from young women and found positive results in $12.7 \%$. A similar study in Tehran-Iran done by Yazdi [25] found the prevalence of Chlamydial infection to be $14.1 \%$ by direct immunoflourescence test. Such a rapid test could be the preferred test in outpatient clinic for rapid detection of Chlamydial antigen.

There are many limitations of the ELISA test especially when testing for uncomplicated genital $C$. trachomatis infection and therefore, it should not be used for screening, because previous chlamydial infections frequently produces long-lasting antibodies that cannot be easily distinguished from the antibodies produced in a current infection. More specific than the ELISA test is the microimmunofluorescence (MIF) test, which uses type specific antigens.

Nucleic acid amplification tests (NAAT's) have generally been more sensitive than traditional tests for the detection of $C$. trachomatis. They also have the advantage that urine can be substituted for the traditional swab specimen, thus reducing the dependence on invasive procedures and expanding the venues where specimens can be obtained.

The frequency of CT infection among women with cervicitis indicates a necessity to implement a routine CT screening program in the major cities. Identification of CT-infected women may prevent its spread, and thereby reduce the high morbidity associated with CT infections among women.

\section{Conclusion}

The results of this study revealed that the commercially available rapid test is of no value in detection of Chlamydia in endocervical swabs. The trend should be toward the substitution of this test by the direct immunoflourescence test. The nucleic acid amplification test which is highly sensitive should be used in future studies to detect the prevalence of Chlamydia infection

\section{References}

[1] S. Kalman, W. Mitchell and R. Marathe et al., Comparative genomes of Chlamydia Pneumoniae and C. trachomatis, Nat Genet 21 (1999) 385-389.

[2] R. C. Brunham, Human immunity to chlamydiae, in: E. S. Stephens (ed.), Chlamydia intracellular biology, pathogenesis, and immunity, Washington, DC: ASM Press, 1999, pp. 211-238.

[3] J. Schachter, Chlamydial infections, N Engl J Med 298 (1978) 428-435, 490-495, 540-549.

[4] M. O. Beem and E. M. Saxon, Respiratory tract colonization and a distinctive pneumonia syndrome in infants with Chlamydia trachomatis, N Engl J Med 163 (1977) 306-310.

[5] B. E. Batteiger and R. B. Jones, Chlamydial infections, Infect Dis Clin North Am; 1 (1987) 55-81.

[6] W. E. Stamm, Diagnosis of Chlamydia trachomatis genitourinary infections, Ann Intern Med 108 (1988) 710-717.

[7] W. Cates and J. N. Wasserheit,. Genital chlamydial infections: epidemiology and reproductive sequelae, Am J Obstst Gynecol 164 (1991) 1771-1781.

[8] G. M. Ryan, S. G. Abdella and V. S. McNeely et al., Chlamydia trachomatis infection in pregnancy and effect of treatment on outcome, Am J Obstet Gynecol 162 (1990) 34-39.

[9] D. A. Scholes, F. E. Stergachis and H. Heidrich, Prevention of pelvic inflammatory disease by screening for cervical chlamydial infection, N Engl J Med 344 (1996) 1362-1366.

[10] H. Thejs, J. Gnarpe and H. Gnarpe et al., Expanded gold standard in the diagnosis of Chlamydia trachomatis in a low prevalence population: diagnostic efficacy of tissue culture, direct immunofluorescence, enzyme immunoassay, PCR and serology, Genitourin Med 70 (1994) 300-303.

[11] Centers for Disease Control and Prevention, Screening tests to detect Chlamydia trachomatis and Neisseria gonorrhoeae infections, MMWR 51(No. RR-15) (2002) 1-39.

[12] R. C. Barnes, Laboratory diagnosis of human chlamydial infections, Clin Microbiol Rev 2 (1989) 119-136.

[13] W. E. Stamm, Chlamydia trachomatis infections: progress and problems, J Infect Dis 179 (suppl 2) (1999) 380-383.

[14] J. M. M. Ossewaarde, M. Rieffe and A. P. M. Rozenburg, et al., Development and clinical evaluation of a polymerase chain reaction test for detection of Chlamydia trachomatis, J Clin Microbiol 30 (1992) 2122-2128.

[15] T. J. Battle, K. L. Golden and J. M. Suchland et al., Evaluation of laboratory testing methods for Chlamydia 
trachomatis infection in the era of nucleic acid amplification tests, J Clin Microbiol 39 (2001) 2924-2927.

[16] S. M. Qadri, J. Akhter and K. Ingacio, Incidence of Chlamydia trachomatis in a large Metropolitan in Saudi Arabia, Saudi Med J 14 (1993) 152-155.

[17] World Health Organization, Sexually transmitted diseases, Press release WHO/64, 25 Aug. 1995.

[18] J. T. Grayston, C. H. Mordhorst and S. P. Wang, Childhood myocarditis associated with Chlamydia trachomatis infection, JAMA 246 (1981) 2823-2827.

[19] D. H. L. Martin, D. A. Koutsky and J. R. Eschenbach et al., Prematurity and perinatal mortality in pregnancies complicated by maternal Chlamydia trachomatis infections, JAMA 247 (1982) 1585-1588.

[20] R. L. Sweet, D. V. Sanders, C. Walker and J. Schachter, Chlamydia trachomatis infection and pregnancy outcome, Am J Obstet Gynecol 156 (1987) 824-833.

[21] H. O. Ghazi, M. H. Daghestani and F. Mohamed, Seropositivity of Chlamydia trachomatis among Saudi pregnant women in Makkah, Journal of Family \& Community Medicine 13 (2) (2006).

[22] F. B. Hashemi, H. B. Pourakbari and J. Z. Yazdi, Frequency of Chlamydia trachomatis in women with cervicitis in Tehran, Iran, Infect Dis Obstet Gynecol. 2007, 67014.

[23] T. Forsey and S. Darougan, Chlamydia Infections in Clinical Tropical Medicine and Communicable DiseasesSexually Transmitted Diseases in The Tropics, London: Bailliere Tindall, 1987, pp. 59-75.

[24] F. Sirmatel, N. Sahin, O. Siramtel and S. Kececi, Chlamydia trachomatis Antigen positivity in women in risk group and it's relationship with the use of antibiotics, JPN.J.INFECT.Dis. 58 (2005) 41-43.

[25] J. Z.Yazdi, M. R. Khorramizadeh, N. Badami, B. Kazemi, F. Aminharati, Z. Eftekhar, A. Berahme and M. Mahmoudi, Comparative assessment of chlamydia trachomatis infection in iranian women with cervicitis: A cross-sectional study, Iranian J Publ Health 35 (2) (2006) $69-75$. 